\title{
ON A PROBLEM OF BANACH
}

\author{
ANDRZEJ PELC AND KAREL PRIKRY ${ }^{1}$
}

\begin{abstract}
Assuming the continuum hypothesis, we obtain a translation invariant version of the following result of $\mathrm{E}$. Grzegorek: There are two countably generated $\sigma$-algebras on the interval $[0,1]$ such that both carry a nonatomic countably additive probability measure, but the $\sigma$-algebra generated by their union does not carry any such measure.
\end{abstract}

Banach [1] asked if there are two countably generated $\sigma$-algebras on the interval $[0,1)$ such that both carry a nonatomic countably additive probability measure, but the $\sigma$-algebra generated by their union does not carry any such measure, i.e. is "nonmeasurable". This problem was answered positively by E. Grzegorek in [2] assuming Martin's Axiom and in [3] without any additional set-theoretic assumptions. The result in [2] satisfies the additional condition that both $\sigma$-algebras extend the $\sigma$-algebra of Borel subsets of $[0,1]$. This additional condition does not hold for the algebras obtained in [3]. Indeed, it is unlikely that such an improvement is possible without some additional set theoretic assumption, since this would imply that there is no real-valued measurable cardinal below the continuum. In the present paper we obtain an analog of the result of [2] in a translation invariant setting - assuming the continuum hypothesis. We do not know whether Martin's axiom suffices.

We shall work on $[0,1)$, mod 1 . The $\sigma$-algebras in the conclusion of the Theorem have as their underlying set the entire $[0,1)$. However, the proof of the Theorem also uses $\sigma$-algebras whose underlying set is a proper subset of $[0,1)$. The concepts we introduce should be viewed within the context of this more general class of $\sigma$-algebras. We say that a $\sigma$-algebra is measurable if it carries a $\sigma$-additive nonatomic probability measure. A $\sigma$-algebra $\Theta$ is translation invariant if for all $A \in \mathbb{Q}$ and $a \in[0,1], A+a \in \mathbb{Q}$. Clearly, $\Theta$ is translation invariant if it is closed under translates of members of a generating subfamily. Hence, the union of two translation invariant $\sigma$-algebras generates a translation invariant $\sigma$-algebra.

Obviously, the underlying set of a translation invariant $\sigma$-algebra has to be the entire $[0,1)$. It will be convenient to work with a less restrictive concept of almost translation invariance. A $\sigma$-algebra $\mathscr{Q}$ is almost translation invariant if for all $A \in \mathcal{A}^{\prime}$ and all $x \in[0,1)$, there is some $B \in \mathbb{Q}$ such that $|(A+x) \triangle B| \leqslant \boldsymbol{\aleph}_{0}$. We shall also call a set $A$ almost translation invariant if for all $x \in[0,1),|(A+x) \triangle A| \leqslant \aleph_{0}$.

Received by the editors July 30, 1982.

1980 Mathematics Subject Classification. Primary 28C10: Secondary 28A05, 04A20.

Kel words and phrases. Translation invariant measure, countably generated $\sigma$-algebra, continuum hypothesis.

${ }^{1}$ The second author received partial support from the NSF Grants MCS 78-01525 and 81-02700

1983 American Mathematical Society $0002-9939 / 83 \$ 1.00+\$ .25$ per page 
The $\sigma$-algebra of the relative Borel subsets of an almost translation invariant set is easily seen to be almost translation invariant. An almost translation invariant $\sigma$-algebra containing all points (and thus all enumerable sets) is translation invariant.

We shall denote the Lebesgue measure by $\lambda$ and the Lebesgue outer measure by $\lambda^{*}$.

Our main result is the following.

TheOREM. Assume the continuum hypothesis. Then there exist countably generated $\sigma$-algebras $\mathscr{Q}_{1}, \mathscr{Q}_{2}$ of subsets of the interval $[0,1)$ and probability measures $\mu_{1}, \mu_{2}$ on $\mathbb{A}_{1}, \mathbb{G}_{2}$, respectively, such that:

(i) $\vec{U}_{1}, \mathbb{U}_{2}$ both contain all Borel sets and are translation invariant;

(ii) $\mu_{1}, \mu_{2}$ both extend the Lebesgue measure and are translation invariant;

(iii) there is no nonatomic probability measure on any $\sigma$-algebra containing $\uplus_{1} \cup \uplus_{2}$.

Lemma $1[4,6]$. Assume $\mathrm{CH}$. Then there is an $X \subset[0,1)$ such that $\lambda^{*}(X)=$ $\lambda^{*}([0,1) \backslash X)=1$ and both $X$ and $[0,1) \backslash X$ are almost translation invariant.

Proof. Let $\left\{x_{\xi}: \xi<\omega_{1}\right\}$ be a Hamel basis for $[0,1)$. Let $Q_{\xi}$ be the subspace spanned by $\left\{x_{\eta}: \eta<\xi\right\}$ (over the rationals) and let $P_{\xi}=Q_{\xi+1} \backslash Q_{\xi}$. Clearly $\left|Q_{\xi}\right| \leqslant \boldsymbol{\aleph}_{0}$. Thus $P_{\xi}$ consists of those elements of $Q_{\xi+1}$ whose representation uses $x_{\xi}$ in a nontrivial way. Hence if $x \in Q_{\xi}$, then $P_{\xi}+x=P_{\xi}$. It now follows easily that for all $A \subseteq \omega_{1}, \cup\left\{P_{\xi}: \xi \in A\right\}$ is almost translation invariant.

Let $\left\{F_{\alpha}: \alpha<\omega_{1}\right\}$ be an enumeration of all closed subsets of $[0,1)$ with $\lambda\left(F_{\alpha}\right)>0$. We can build inductively $A \subset \omega_{1}$ such that for all $\alpha \in \omega_{1}$, there are $\xi \in A$, $\eta \in \omega_{1} \backslash A$ such that both $P_{\xi} \cap F_{\alpha}$ and $P_{\eta} \cap F_{\alpha}$ are not empty. Now $X=$ $\cup\left\{P_{\xi}: \xi \in A\right\}$ as is required.

Lemma 2 [5]. Assume CH. Let $\mathscr{F} \subset \mathcal{P}([0,1)),|\mathcal{F}|=\boldsymbol{\aleph}_{1}$. Then there exists a countably generated $\sigma$-algebra $\mathbb{Q}$ on $[0,1)$ such that $\mathscr{F} \subset \mathbb{Q}$.

Proof. We can suppose $\mathscr{F} \subset \mathcal{P}\left(\omega_{1}\right), \mathscr{F}=\left\{F_{\alpha}: \alpha<\omega_{1}\right\}$. Set

$$
F=\cup\left\{\{\alpha\} \times F_{\alpha}: \alpha<\omega_{1}\right\} .
$$

Now by [5,7], $F$ belongs to the $\sigma$-algebra generated by some sets $A_{n} \times B_{n}, n \in \omega$, where $A_{n}, B_{n} \subset \omega_{1}$. It is easy to see that $\mathcal{F}_{\text {in }}$ included in the $\sigma$-algebra generated by the $B_{n}$ 's.

Lemma 3. Assume $\mathrm{CH}$. Then for every uncountable $X \subset[0,1)$, there is a nonmeasurable countably generated $\sigma$-algebra $@$ on $X$ containing all Borel subsets of $X$.

Proof. By [7], there is an $L \subset[0,1)$ such that $|L|=\boldsymbol{\aleph}_{1}$ and $|L \cap N| \leqslant \boldsymbol{\aleph}_{0}$ for every meager $N$. It follows easily from the fact that every Borel measure on $[0,1)$ concentrates on a meager set that the $\sigma$-algebra of Borel subsets of $L$ is nonmeasurable.

Since the properties involved are preserved under bijections, the $\sigma$-algebra constructed on $L$ can be transplanted to any set $X \subset[0,1)$ of cardinality $\boldsymbol{\aleph}_{1}$. The resulting $\sigma$-algebra contains singletons and augmented by adding the Borel subsets of $X$ remains nonmeasurable. 
Proof of the Theorem. Let $X$ be as in Lemma 1 and $X^{\prime}=[0,1) \backslash X$. Let $\Re_{0}$ be a nonmeasurable countably generated $\sigma$-algebra on $X$ containing the Borel subsets of $X$ (see Lemma 3). Let $T\left(\mathfrak{N}_{0}\right)=\left\{(x+S) \cap X: x \in[0,1), S \in \mathfrak{N}_{0}\right\}$. Then $\left|T\left(\mathfrak{x}_{0}\right)\right|$ $=\boldsymbol{\aleph}_{1}$, and thus by Lemma 2 , there is a countably generated $\sigma$-algebra $\Re_{1} \supset T\left(\Re_{0}\right)$. Defining $T\left(\Re_{1}\right)$ analogously to $T\left(\Re_{0}\right)$, we can find countably generated $\Re_{2} \supset$ $T\left(\Re_{1}\right)$, and so on. Let $\Re$ be the $\sigma$-algebra generated by $\cup\left\{\mathscr{N}_{n}: n \in \omega\right\}$. Then $\mathfrak{x}$ is countably generated and nonmeasurable. It also follows easily from the almost translation invariance of $X$ that $\gamma$ is almost translation invariant. Let $\Re{ }^{\prime}$ be obtained analogously on $X^{\prime}$ and let $\mathfrak{M}$ and $\mathfrak{N}^{\prime}$ be the $\sigma$-algebras of Borel subsets of $X$ and $X^{\prime}$, respectively. Thus $\mathscr{N}$ and $\mathscr{N}^{\prime}$ are almost translation invariant and so are $\mathbb{Q}_{1}=\left\{A \cup A^{\prime}: A \in \mathfrak{N}, A^{\prime} \in \mathcal{N}^{\prime}\right\}$ and $\mathbb{Q}_{2}=\left\{A \cup A^{\prime}: A \in \mathfrak{N}, A^{\prime} \in \mathcal{N} \mathcal{R}^{\prime}\right\}$. But $\mathbb{U}_{1}$ and $\mathcal{Q}_{2}$ contain all points, hence, they are translation invariant.

We now define $\mu_{i}$ on $\mathcal{Q}_{i}(i=1,2)$, as follows. Let $A \cup A^{\prime} \in \mathcal{Q}_{1}$ where: $A \in \mathbb{M}$, $A^{\prime} \in \mathcal{N}^{\prime}$. Set $\mu_{1}\left(A \cup A^{\prime}\right)=\lambda^{*}(A)$. For $A \cup A^{\prime} \in \mathbb{Q}_{2}$ where $A \in \mathscr{N}, A^{\prime} \in \mathfrak{M} \Re^{\prime}$, we set $\mu_{2}\left(A \cup A^{\prime}\right)=\lambda^{*}\left(A^{\prime}\right)$. (i) and (ii) now easily follow. (We remark that $\lambda^{*}$, restricted to the Borel subsets of an arbitrary set of outer measure one, is a countably additive probability measure.)

Finally, let $\mathcal{Q}^{\mathcal{B}}$ be the least $\sigma$-algebra containing $\mathbb{Q}_{1} \cup \mathbb{Q}_{2}$. If $\mu$ is a nonatomic probability measure on $\mathbb{Q}$, then either $\mu(X)>0$ or $\mu\left(X^{\prime}\right)>0$. Without loss of generality let $\mu(X)>0$. Since $\mathfrak{N} \subset \mathbb{Q}$ and $X \in \mathfrak{N}, \mu$ restricted to $\mathscr{N}$ is a nonatomic finite nontrivial measure on $\mathcal{X}$. This is a contradiction since $\mathscr{N}$ is nonmeasurable. Hence, (iii) holds and the Theorem is proved.

\section{REFERENCES}

1. S. Banach, Sur les suites d'ensembles excluant l'existence d' une mesure (Note posthume avec préface et commentaire de E. Marczewski), Colloq. Math. 1 (1948), 103-108.

2. E. Grzegorek, Remarks on o-fields without continuous measures, Colloq. Math. 39 (1978), 73-75.

3. Solution of a problem of Banach on $\sigma$-fields without continuous measure. Bull. Acad. Polon. Sci. Sér. Sci. Math. 28 (1980), 7-10.

4. S. Kakutani and J. Oxtoby, Construction of a non-separable invariant extension of the Lebesgue measure space, Ann. of Math. (2) 52 (1950), 580-590.

5. B. V. Rao, On discrete Borel spaces and projective sets, Bull. Amer. Math. Soc. 75 (1969), 614-617.

6. W. Sierpiński, Sur les translations des ensembles linéaires, Fund. Math. 19 (1932), 22-28.

7. H. Hyothese du continu. Chelsea, New York, 1956.

Department of Mathematics, Warsaw University, Warsaw, Poland

Department of Mathematics, University of Minnesota, Minneapolis, Minnesota 55455 